\title{
Uniqueness and comparison properties of the viscosity solution to some singular HJB equations
}

\author{
Anna Lisa AMADORI \\ Dipartimento di Scienze Applicate \\ Università di Napoli "Parthenope" \\ Via A. De Gasperi, 5 \\ 80133 Napoli, Italy \\ e-mail: annalisa.amadori@uniparthenope.it
}

\begin{abstract}
We study viscosity solutions to a class of HJB equations with singular coefficients near at the boundary: cases with either vanishing, or oscillating, or blowing-up diffusion coefficients are included. Because of proper structural conditions, strong comparison principle holds without assigning spatial boundary data, and unbounded initial data can be handled. The result applies to stochastic models for interest rate, and yields new results concerning Cauchy problems with unbounded coefficients.
\end{abstract}

2000 Mathematics Subject Classification: 49L25, 35K65, 35R25.

Key words: HJB equation, viscosity solutions, degenerate and unbounded coefficients, CIR model for interest rate.

\section{Introduction}

We consider the finite horizon minimization problem

$$
\begin{array}{r}
\mathbf{U}(x, t)=\inf _{\left(\alpha_{s}\right)_{s}} \mathbb{E}_{x t}\left[\int_{t}^{T} e^{-\int_{s}^{T} r\left(X_{v}, v, \alpha_{v}\right) d v} f\left(X_{s}, s, \alpha_{s}\right) d s\right. \\
\left.+e^{-\int_{t}^{T} r\left(X_{v}, v, \alpha_{v}\right) d v} u_{o}\left(X_{T}\right)\right],
\end{array}
$$

for some controlled degenerate diffusion in an open domain $\Omega \subset \mathbb{R}^{\mathrm{N}}$ :

$$
\left\{\begin{array}{l}
d X_{s}=b\left(X_{s}, s, \alpha_{s}\right) d s+\sigma\left(X_{s}, s, \alpha_{s}\right) d W_{s}, \quad s>t, \\
X_{t}=x \in \Omega .
\end{array}\right.
$$


Here $\left(W_{t}\right)_{t}$ is a D-dimensional Brownian motion (with possibly $\mathrm{D} \leq \mathrm{N}$ ) and the control $\left(\alpha_{t}\right)$ is any adapted process with values in a compact set $\mathcal{A} \subset \mathbb{R}^{\mathrm{P}}$. Moreover $\sigma, b, f$, and $r$ are continuous functions of $x \in \Omega, t \geq 0, \alpha \in \mathcal{A}: \sigma$ takes values in the set of $\mathrm{N} \times \mathrm{D}$ real matrices, $b$ in $\mathbb{R}^{\mathrm{N}}$, and $f, r$ are real valued.

It is well-known that the related Hamilton-Jacobi-Bellman equation reads

$$
-\partial_{t} u+H\left(x, t, u, D u, D^{2} u\right)=0, \quad x \in \Omega, t \in[0, T),
$$

where

$$
\begin{aligned}
& H(x, t, u, p, X) \\
& \quad=\sup _{\alpha \in \mathcal{A}}\left\{-\frac{1}{2} \operatorname{tr}\left[\sigma \sigma^{T}(x, t, \alpha) X\right]-b(x, t, \alpha) p+r(x, t, \alpha) u-f(x, t, \alpha)\right\},
\end{aligned}
$$

for any $x \in \Omega, t \in[0, T), u \in \mathbb{R}, p \in \mathbb{R}^{\mathrm{N}}$ and $X$ in the set of real $\mathrm{N} \times \mathrm{N}$ matrices. Such partial differential equation is endowed with a final condition

$$
u(x, T)=u_{o}(x), \quad x \in \Omega .
$$

The classical stochastic control problem (1.1)-(1.2) and its relationship with viscosity solutions to the related HJB equation (1.3)-(1.4) have been extensively studied; a wide literature arose on the topic, and we only mention $[16,12,1,18]$. A standard assumption in viscosity theory is that the coefficients $\sigma_{i j}, b_{i}$ are Lipschitz continuous w.r.t. the $x$-variable in the closed set $\bar{\Omega}$. In this paper we allow the coefficients to be singular near at the boundary $\partial \Omega$. In dimension one, let us take $\Omega=(0, \infty)$ : near at the boundary $x=0$, we suppose that there is a constant $\mathrm{C}$ so that

$$
0<\frac{1}{2} \sigma^{2} \leq \frac{x}{1-\mathrm{C} x} b
$$

for all $t \in[0, T], \alpha \in \mathcal{A}$ and $x$ near at 0 .

The following non-standard items are included: i) $\sigma$ vanishes with rate $x^{\theta}, \theta<1$, ii) $\sigma$ oscillates and $b$ blows up, iii) both $\sigma$ and $b$ blow up. Concerning multidimensional case, the structure condition (1.5) generalizes by substituting $x$ with the distance to the boundary if $\Omega$ is smooth (see later on condition (2.1)). Otherwise, if $\Omega$ has corners, it is assumed that (1.5) hold in any space direction (see later on condition (2.5) for precise details). Our technique adapts to handle the case where $\Omega$ is all the space $\mathbb{R}^{N}$ and the volatility $\sigma$ is unbounded: we defer to Section 3 the statement of assumptions and results.

The interest for this kind of diffusions is motivated by the so called Cox-Ingersoll-Ross model for interest rate [5]:

$$
\left\{\begin{array}{l}
d X_{t}=k\left(\mathrm{M}-X_{t}\right) d t+\sigma_{o} \sqrt{X_{t}} d W_{t}, \quad t>0, \\
X_{0}=x>0 .
\end{array}\right.
$$


Here $\left(W_{t}\right)_{t}$ is a standard Brownian motion, $\mathrm{M} \geq 0$ stands for the asymptotic mean, $k \geq 0$ stands for the reversion speed, and $\sigma_{o}^{2} X$ is the variance. In practice the interest rate $X$ is a positive defined quantity: this fact is plugged into the model by imposing that

$$
0<\frac{1}{2} \sigma_{o}^{2} \leq k \mathrm{M}
$$

which is equivalent to our assumption (1.5) in this particular case. It is easily checked that, under this condition, the set $(0,+\infty)$ is invariant for the process $\left(X_{t}\right)_{t}$, i.e. the first exit time from $(0,+\infty)$ is infinite with probability 1 (see, for instance, $[15, \S 6$, Proposition 2.4]). Multi-dimensional models arise in forward rate modeling, as empirical evidence suggests that all the forward curve can be reconstructed starting by a finite number of representative interest rates described by a processes of type (1.6). We refer to [2] and the references therein for a detailed discussion about this topic. In this case the invariant set is the positive cone $(0, \infty)^{\mathrm{N}}$, which does not have smooth boundary.

The main goal of this paper is to characterize the value function (1.1) by means of the related Hamilton-Jacobi-Bellman equation (1.3), endowed with some additional condition at the parabolic boundary. Since $\sigma$ fails to be Lipschitz continuous with respect to $x$ on $\bar{\Omega}$, the standard theory of viscosity solutions does not apply. Indeed, comparison results including HJB equations with merely Holder continuous coefficients are available in the literature, see [13, Theorems II.2, III.1]. They assume a-priori that the sub/supersolutions are Lipschitz continuous inside $\Omega$ and ordered on the boundary $\partial \Omega$, and apply when the Holder exponent is greater than $1 / 2$; moreover Theorem III.1 applies to strictly elliptic equations. Our point of view is different, because we address equations that show a particular structure condition at the boundary. So we do not ask sub/supersolutions to be regular, nor impose some restriction to the Holder exponent, but we rather investigate which additional condition has to be imposed in the boundary to get uniqueness. Following a former idea by Feller, we do not prescribe any Dirichlet or Neumann condition, but we merely fix a critical blow up rate (see later on (2.2) or (2.7), respectively), that we show to be optimal for comparison. We establish a strong comparison principle among semicontinuous sub and supersolutions which possibly blow up near at the side boundary, by combining standard viscosity with barrier arguments. As a by-product, the value function (1.1) is characterized as the unique viscosity solution to the HJB equation (1.3)-(1.4).

The uncontrolled case, which corresponds to the case where $\mathcal{A}$ is a singleton and gives rise to a linear PDE, has been intensively studied. In the 50's Feller studied the one dimensional example (1.6), see [9-11]. He pointed out that the process $\left(X_{t}\right)_{t}$ never hits the boundary $x=0$, hence well-posedness for the related deterministic equation holds without assigning neither a Dirichlet, or a Neumann type boundary condition. More recently these problems have been attacked by means of spectral analysis technique; for instance the paper [3] establishes a sufficient condition for well posedness in the class of classical solutions satisfying a 
proper regularity condition near at the boundary. Problems presenting a boundary singularity of type (1.5) can be carried to Cauchy problems with unbounded coefficients, which have been studied, among others, by [14], [8], and [17]. These works present different well posedness results in the class of vanishing or bounded functions. The present result, when applies, allows to deal with unbounded functions, and to avoid non-degeneracy assumptions inside the domain.

This paper is organized as follows. In Section 2 we list the assumptions and the main results, and we provide examples and counterexamples. In Section 3 we illustrate how the obtained results can be adapted to Cauchy problems with unbounded coefficients, while Section 4 is devoted to the proof of the comparison principles.

\section{Assumptions and main result}

In order to list the precise definitions and assumptions that shall be in use, we introduce some notations. Let $d(x)$ denote the function distance to the boundary $\partial \Omega$ :

$$
d(x):=\inf \{|x-\bar{x}|: \bar{x} \in \partial \Omega\} .
$$

For any $t \in[0, T]$ and $\alpha \in \mathcal{A}$ we define the linear degenerate elliptic operator

$$
\mathcal{L}_{t \alpha} \varphi(x):=-\frac{1}{2} \operatorname{tr}\left[\sigma \sigma^{T}(x, t, \alpha) D^{2} \varphi(x)\right]-b(x, t, \alpha) \cdot D \varphi(x) .
$$

For any $\ell \geq 0$ we introduce the function

$$
h_{\ell}(s):= \begin{cases}\max \left(\log \frac{1}{s}, 1\right) & \text { if } \ell=0, \\ \max \left(\frac{1}{s^{\ell}}, 1\right) & \text { elsewhere. }\end{cases}
$$

In the following we shall adopt the standard notion of viscosity solutions. By a viscosity subsolution to (1.3)-(1.4) we mean a function $u$ which

(i) is upper semicontinuous in the set $\Omega \times[0, T]$,

(ii) solves the partial differential inequality $-\partial_{t} u+H\left(x, t, u, D u, D^{2} u\right) \leq 0$ in the viscosity sense according to [6, Definition 2.2], in the set $\Omega \times[0, T)$,

(iii) verifies pointwise the inequality $u(x, T) \leq u_{o}(x)$, in the set $\Omega \times\{T\}$.

The definition of supersolution is perfectly symmetrical; a solution is any function whose upper semicontinuous envelope is a subsolution, and whose lower semicontinuous envelope is a supersolution. It is worst remarking that no condition is imposed in the side boundary $\partial \Omega \times[0, T)$ : neither on the value of the function $u$, nor on its partial derivatives. 
The standing assumptions about the data of the control problem will be:

Assumption 1. For $\psi=\sigma_{i j}, b_{i}, f, r$ (with $i=1, \cdots, \mathrm{N}, j=1, \cdots, \mathrm{D}$ ) the following hold: $\psi$ is continuous on $\Omega \times[0, T] \times \mathcal{A}$; for any $\rho>0, t \in[0, T]$, and $\alpha \in \mathcal{A}$, the function $\psi(\cdot, t, \alpha)$ is Lipschitz continuous on the set $\{x \in \Omega: d(x) \geq \rho\}$ with

$$
\sup \left\{\|\psi\|_{\mathcal{C}^{0,1}(\{x \in \Omega: d(x) \geq \rho\})}: t \in[0, T], \alpha \in \mathcal{A}\right\}<\infty .
$$

Moreover the function $r$ is bounded from below on $\Omega \times[0, T] \times \mathcal{A}$, and the function $u_{o}$ is continuous on $\Omega$.

Next we state separate hypotheses depending on whether the domain $\Omega$ has smooth boundary or not.

\section{Case a: the domain has smooth boundary.}

In the first case we suppose that

Assumption 2. The domain $\Omega$ is an open, bounded and connected subset of $\mathbb{R}^{\mathrm{N}}$ with boundary $\partial \Omega$ of class $W^{3, \infty}$. For any $\bar{x} \in \partial \Omega$ we have

$$
\limsup _{\Omega \ni x \rightarrow \bar{x}} \sup _{\substack{t \in[0, T] \\ \alpha \in \mathcal{A}}}\left(\frac{1}{d}+\frac{\mathcal{L}_{t \alpha} d(x)}{\frac{1}{2}\left|\sigma^{T} D d\right|^{2}}\right)<\infty .
$$

Assumption 2 is sufficient to deal with bounded solutions. One can deal with unbounded solutions if condition (2.1) holds uniformly with respect to $\bar{x} \in \partial \Omega$, namely

Assumption 3. There exist $\rho>0$ and $\ell \geq 0$ so that the function

$$
(x, t, \alpha) \mapsto \frac{1+\ell}{d}+\frac{\mathcal{L}_{t \alpha} d}{\frac{1}{2}\left|\sigma^{T} D d\right|^{2}}
$$

is bounded from above in the set $\{x \in \Omega: d(x) \leq \rho\} \times[0, T] \times \mathcal{A}$.

Our main results concerning smooth domains state as follows.

Theorem 1 (Characterization of the value function) Assume 1 and 2.

(i) If $f$ and $u_{o}$ are bounded, then the function $\mathbf{U}$ defined by (1.1) is the unique bounded viscosity solution to (1.3)-(1.4).

(ii) Let also Assumption 3 holds, and suppose that there are $0 \leq \kappa<\ell$ and $\rho>0$ such that the function

$$
(x, t, \alpha) \mapsto \frac{|f|+\left|u_{o}\right|}{h_{\kappa}(d)}
$$


is bounded from above in the set $\{x \in \Omega: d(x) \leq \rho\} \times[0, T] \times \mathcal{A}$. Then the value function $\mathbf{U}$ is the unique viscosity solution to (1.3)-(1.4) satisfying the blow-up condition

$$
\lim _{\Omega \ni x \rightarrow \bar{x}} \sup _{t \in[0, T]} \frac{|\mathbf{U}|}{h_{\ell}(d)}=0 \quad \text { for every } \bar{x} \in \partial \Omega .
$$

The value function $\mathbf{U}$ solves (1.3) by the dynamic programming principle (see, for instance, [16, Theorem I.1]), and satisfies the final condition (1.4) by construction. By following the line of analogous computations in [18, §4, Proposition 3.1 , one can check that the growth of the value function $\mathbf{U}$ near at the boundary $\partial \Omega$ is controlled by $h_{\kappa}(d)$, and therefore condition (2.2) follows. Uniqueness of viscosity solutions comes from the next strong comparison result.

Theorem 2 (Comparison principle) Assume 1, 2, and take $u$ and $v$ respectively an u.s.c. subsolution and a l.s.c. supersolution to (1.3) with

$$
u \leq v \quad \text { on } \Omega \times\{T\} .
$$

(i) If $u$ and $v$ are bounded, then $u \leq v$ in $\Omega \times[0, T]$.

(ii) If also Assumption 3 holds, and $u$ and $v$ are locally bounded in $\Omega \times[0, T]$ with

$$
\limsup _{\Omega \ni x \rightarrow \bar{x}} \sup _{t \in[0, T]} \frac{u}{h_{\ell}(d)} \leq 0, \quad \liminf _{\Omega \ni x \rightarrow \bar{x}} \inf _{t \in[0, T]} \frac{v}{h_{\ell}(d)} \geq 0 \quad \text { for all } \bar{x} \in \partial \Omega,
$$

$$
\text { then } u \leq v \text { in } \Omega \times[0, T] \text {. }
$$

\section{Case b: the domain has corners.}

We take for simplicity a sample domain with corners and unbounded, which is of interest by itself in applications to interest rate models. We leave to the reader the straightforward extension to domains with boundary piecewise $W^{3, \infty}$.

Assumption 4. The domain $\Omega$ is the positive orthant $(0, \infty)^{\mathrm{N}}$. For any $\bar{x} \in \partial \Omega$ and $i=1, \cdots, \mathrm{N}$ so that $\bar{x}_{i}=0$ we have

$$
\limsup _{\Omega \ni x \rightarrow \bar{x}} \sup _{\substack{t \in[0, T] \\ \alpha \in \mathcal{A}}}\left(\frac{1}{x_{i}}-\frac{b \cdot e_{i}}{\frac{1}{2}\left|\sigma^{T} e_{i}\right|^{2}}\right)<\infty .
$$

Here $x_{i}$ stands for the $i$-th component of the vector $x$, and $e_{i}$ for the unit vector in the $i$-th direction. Also in this case, we state an uniform version of condition (2.5) that matches unbounded data. 
Assumption 5. There exist $\rho>0$ and $\ell_{1}, \cdots, \ell_{\mathrm{N}} \geq 0$ so that the functions

$$
(x, t, \alpha) \mapsto \frac{1+\ell_{i}}{x_{i}}-\frac{b \cdot e_{i}}{\frac{1}{2}\left|\sigma^{T} e_{i}\right|^{2}}
$$

are bounded from above in the set $\{x \in \Omega: d(x) \leq \rho\} \times[0, T] \times \mathcal{A}$, for all $i=1, \cdots$, N.

For future convenience, for any $\bar{x} \in \partial \Omega$ we introduce the set of indexes

$$
\mathrm{I}(\bar{x}):=\left\{i \in\{1, \cdots, \mathrm{N}\}: \bar{x}_{i}=0\right\} .
$$

Our main results in the non-smooth case are

Theorem 3 (Characterization of the value function) Assume 1 and 4 .

(i) If the functions $f$ and $u_{o}$ have global sub-quadratic growth, i.e. there exists $\gamma<2$ so that the function

$$
(x, t, \alpha) \mapsto \frac{\left|u_{o}\right|+|f|}{1+|x|^{\gamma}}
$$

is bounded in $\Omega \times[0, T] \times \mathcal{A}$, then the function $\mathbf{U}$ defined by (1.1) is the unique viscosity solution to (1.3)-(1.4) such that

$$
\sup _{\Omega \times[0, T]} \frac{\mathbf{U}}{1+|x|^{\gamma}}<\infty .
$$

(ii) Let Assumption 5 holds, and suppose that there are $\gamma<2,0 \leq \kappa_{i}<\ell_{i}$ and $\mathrm{R}, \rho>0$ such that the functions

$$
(x, t, \alpha) \mapsto \frac{|f|+\left|u_{o}\right|}{|x|^{\gamma}}
$$

and

$$
(x, t, \alpha) \mapsto \frac{|f|+\left|u_{o}\right|}{\sum_{i=1}^{N} h_{\kappa_{i}}\left(x_{i}\right)}
$$

are bounded from above respectively in the set $\{x \in \Omega:|x| \geq \mathrm{R}\} \times[0, T] \times \mathcal{A}$ and $\{x \in \Omega: d(x) \leq \rho\} \times[0, T] \times \mathcal{A}$. Then the value function $\mathbf{U}$ is the unique viscosity solution to (1.3)-(1.4) satisfying the blow-up conditions

$$
\begin{aligned}
& \limsup _{|x| \rightarrow \infty} \sup _{t \in[0, T]} \frac{|\mathbf{U}|}{|x|^{2}}=0 \\
& \lim _{\Omega \ni x \rightarrow \bar{x}} \sup _{t \in[0, T]} \frac{|\mathbf{U}|}{\sum_{i \in \mathrm{I}(\bar{x})} h_{\ell_{i}}\left(x_{i}\right)}=0 \quad \text { for every } \bar{x} \in \partial \Omega .
\end{aligned}
$$


Theorem 4 (Comparison principle) Assume 1, 4, and take $u$ and $v$ respectively an u.s.c., locally bounded subsolution and a l.s.c., locally bounded supersolution to (1.3) with

$$
u \leq v \quad \text { on } \Omega \times\{T\} .
$$

(i) If there is $\gamma<2$ such that

$$
\sup _{\Omega \times[0, T]} \frac{u}{1+|x|^{\gamma}}<\infty, \quad \inf _{\Omega \times[0, T]} \frac{v}{1+|x|^{\gamma}}>-\infty,
$$

then $u \leq v$ in $\Omega \times[0, T]$.

(ii) If also Assumption 5 holds and

$$
\begin{array}{rr}
\limsup _{|x| \rightarrow \infty} \sup _{t \in[0, T]} \frac{u}{|x|^{2}} \leq 0, & \liminf _{|x| \rightarrow \infty} \inf _{t \in[0, T]} \frac{v}{|x|^{2}} \geq 0 \\
\limsup _{\Omega \ni x \rightarrow \bar{x}} \sup _{t \in[0, T]} \frac{u}{\sum_{i \in \mathrm{I}(\bar{x})} h_{\ell_{i}}\left(x_{i}\right)} \leq 0, & \liminf _{\Omega \ni x \rightarrow \bar{x}} \inf _{t \in[0, T]} \frac{v}{\sum_{i \in \mathrm{I}(\bar{x})} h_{\ell_{i}}\left(x_{i}\right)} \geq 0
\end{array}
$$

for all $\bar{x} \in \partial \Omega$, then $u \leq v$ in $\Omega \times[0, T]$.

Remark 1 The structural condition (2.1) (respectively, (2.5)) reduces to (1.5) if $\mathrm{N}=1$. If we stand in case $\mathrm{b}$ and $\bar{x} \in \partial \Omega$, any direction indexed by $i \in \mathrm{I}(\bar{x})$ is nontangential to the boundary. If we take a point $\bar{x}$ in a smooth part of the boundary, $\mathrm{I}(\bar{x})$ is a singleton; it is easily seen that the property (2.5) and the Assumption 5 become (2.1) and 3, respectively. Otherwise, let us take the point $\bar{x}$ in a hedge. Now the function distance to the boundary is not differentiable in classical sense, but its "generalized derivative" is the cone generated by that directions indexed by $i \in \mathrm{I}(\bar{x})$. For this reason (2.5) could be the natural way to read (2.1) in the framework of non-smooth analysis (see, for instance, [4]).

Remark 2 Concerning the behavior of data for large $|x|$, we restrict the analysis to sub-quadratic growth at infinity in order to put into light the main new idea. After making suitable assumptions on the behavior of $\sigma, b, f$ for large $|x|$, one can handle polynomial growth by following the line of [7]. Otherwise, if $\sigma$ itself is unbounded, we refer to Section 3 for a detailed treatment of the growth at infinity.

We devote the last part of this section to some linear examples and counterexamples in dimension one. The first example shows that, if condition (2.1) (respectively, (2.5)) is dropped, comparison does not hold even between bounded sub/supersolutions. Moreover the blow-up condition (2.4) (respectively, (2.11)) can not be removed. 
Example 1 Let us take a linear one-dimensional problem, with diffusion vanishing at the boundary and merely Holder continuous:

$$
\left\{\begin{array}{lr}
-\partial_{t} u=x \partial_{x x}^{2} u+(\mathrm{M}-x) \partial_{x} u, & x>0, t \in[0,2), \\
u(x, 2)=0, & x>0 .
\end{array}\right.
$$

Problem (2.12) corresponds to (1.3) with no control, $\sigma=\sqrt{2 x}, b=\mathrm{M}-x, f=$ $r=u_{o}=0$. Condition (2.1) (equivalently, (2.5)) holds if and only if $\mathrm{M} \geq 1$. Concerning the maximal blow up rate we have

$$
\frac{1+\ell}{d}+\frac{\mathcal{L}_{t \alpha} d}{\frac{1}{2}\left|\sigma^{T} D d\right|^{2}}=\frac{1+\ell}{x}-\frac{b}{\frac{1}{2}|\sigma|^{2}}=\frac{1+\ell-\mathrm{M}}{x}+1,
$$

hence the optimal parameter is $\ell=\mathrm{M}-1$.

Trivially $u=0$ solves this problem, after we set

$$
\underline{u}(x, t)= \begin{cases}\int_{\frac{x}{1-t}}^{1} e^{-\xi} \xi^{-\mathrm{M}} d \xi & \text { if } t \in[0,1) \text { and } x<1-t \\ 0 & \text { elsewhere. }\end{cases}
$$

The function $\underline{u}$ has positive supremum and is a viscosity subsolution to (2.12). Indeed it is easily seen that it is a subsolution in classical sense at $x \neq 1-t$, while it has empty upper semi-jet at $x=1-t$.

If $\mathrm{M}<1$ (equivalently $\ell<0$ ), $\underline{u}$ is bounded: because hypothesis $(2.1)$ (equivalently, $(2.5))$ is not fulfilled, comparison does not hold even in the class of bounded sub/supersolutions. It is worth mentioning that the underlying stochastic process, i.e. the solution to the stochastic differential equation (1.2), becomes negative in finite time with positive probability.

On the contrary, if $\mathrm{M} \geq 1$ (equivalently $\ell \geq 0$ ), $\underline{u}$ has critical blow up rate. In other words, conditions (2.4) and (2.11) are sharp.

As mentioned in the introduction, other comparison principles that apply to merely Holder continuous diffusions require that the Holder exponent is greater than $1 / 2$. On the contrary, Theorems 2 and 4 applies also if the outwards component of the diffusion $\sigma^{T} D d$ (respectively, $\sigma^{T} e_{i}$ ) vanishes at the boundary steeper than $\sqrt{d}$ (respectively, $\sqrt{x_{i}}$ ). It has to be stressed that, in this case, both conditions (2.1) and (2.5) imply that the drift $b$ has to blow up. It is necessary in order to have well posedness without imposing any boundary datum. The problem, indeed, can be well posed if the drift coefficient $b$ blows up, and ill posed if $b$ is smooth. 
Example 2 Take the linear one-dimensional problem

$$
\left\{\begin{array}{lr}
-\partial_{t} u=\sqrt{x} \partial_{x x}^{2} u+\left(\frac{\beta}{\sqrt{x}}-\frac{2}{3} x\right) \partial_{x} u, & x>0, t \in[0,2), \\
u(x, 2)=0, & x>0 .
\end{array}\right.
$$

After the change of variable $\tilde{x}=4 x^{\frac{3}{2}} / 9$, problem (2.13) reduces to (2.12) with $\mathrm{M}=(1+2 \beta) / 3$. Then the same arguments of Example 1 yields that it has an unique solution only if $\beta \geq 1$. In particular, the coefficient of the first order term has to blow up at $x=0$. If $\beta=0$, the same coefficient stays bounded, but comparison does not hold.

Finally, we apply our result to a problem with exploding coefficients.

Example 3 We consider here

$$
\left\{\begin{array}{lr}
-\partial_{t} u=\frac{1}{x} \partial_{x x}^{2} u+\left(\frac{\beta}{x^{2}}-\frac{1}{3} x\right) \partial_{x} u, & x>0, t \in[0,2), \\
u(x, 2)=0, & x>0 .
\end{array}\right.
$$

After the change of variable $\tilde{x}=x^{3} / 9$, problem (2.14) reduces to (2.12) with $\mathrm{M}=(2+\beta) / 3$. Then the same arguments of Example 1 yields that it has an unique solution whenever $\beta \geq 1$.

\section{Application to Cauchy problem with unbounded coefficients}

The comparison result stated by Theorem 4 can be used to obtain well posedness for Cauchy problems with unbounded coefficients, which have been studied by several authors in recent years.

We consider here the HJB equation (1.3) in all the space and take

Assumption 6. The domain $\Omega$ is all $\mathbb{R}^{\mathrm{N}}$. The real functions $\sigma_{i j}, b_{i}, f$, and $r$ are continuous on $\mathbb{R}^{\mathrm{N}} \times[0, T] \times \mathcal{A}$, and locally Lipschitz continuous in the variable $x$, uniformly w.r.t. $t \in[0, T]$ and $\alpha \in \mathcal{A}$. Moreover $r$ is bounded from below and the function $u_{o}$ is continuous in $\mathbb{R}^{\mathrm{N}}$.

For all $i=1, \cdots, \mathrm{N}$, there exist $\lambda_{i}>0$ such that

$$
\begin{aligned}
& \liminf _{|x| \rightarrow \infty} \inf _{\substack{t \in[0, T] \\
\alpha \in \mathcal{A}}} \frac{b \cdot e_{i}}{\frac{1}{2}\left|\sigma^{T} e_{i}\right|^{2}} e^{-\lambda_{i} x_{i}}>-\infty, \\
& \limsup _{|x| \rightarrow \infty} \sup _{\substack{t \in[0, T] \\
\alpha \in \mathcal{A}}} \frac{b \cdot e_{i}}{\frac{1}{2}\left|\sigma^{T} e_{i}\right|^{2}} e^{\lambda_{i} x_{i}}<\infty .
\end{aligned}
$$


Concerning the maximal growth rate, by assumption there exist $\mathrm{N}$ parameters $\ell_{i} \geq 0$ and $R>0$ so that the functions

$$
\begin{aligned}
& (x, t, \alpha) \mapsto\left(\ell_{i}-\frac{b \cdot e_{i}}{\frac{1}{2}\left|\sigma^{T} e_{i}\right|^{2}}\right) e^{-\lambda_{i} x_{i}}, \\
& (x, t, \alpha) \mapsto\left(\ell_{i}+\frac{b \cdot e_{i}}{\frac{1}{2}\left|\sigma^{T} e_{i}\right|^{2}}\right) e^{\lambda_{i} x_{i}}
\end{aligned}
$$

are bounded from above in the set $\{|x| \geq R\} \times[0, T] \times \mathcal{A}$.

After a suitable change of variable, we obtain the following comparison result:

Theorem 5 (Comparison principle - unbounded coefficients) Assume 6 and let $u$ and $v$ be respectively an u.s.c., locally bounded subsolution and a l.s.c., locally bounded supersolution to (1.3) with

$$
\begin{array}{rlrl}
u & \leq v & \text { on } \mathbb{R}^{\mathrm{N}} \times\{T\}, \\
\limsup _{|x| \rightarrow \infty} \sup _{t \in[0, T]} \frac{u}{\sum_{i=1}^{\mathrm{N}} k_{\ell_{i}}\left(\left|x_{i}\right|\right)} \leq 0, \quad \liminf _{|x| \rightarrow \infty} \inf _{t \in[0, T]} \frac{v}{\sum_{i=1}^{\mathrm{N}} k_{\ell_{i}}\left(\left|x_{i}\right|\right)} \geq 0,
\end{array}
$$

where $\ell_{1}, \cdots, \ell_{N}$ are the nonnegative parameters appearing in (3.2). Then $u \leq v$ in $\mathbb{R}^{\mathrm{N}} \times[0, T]$.

Here we have used the weight function

$$
k_{\ell}(s):= \begin{cases}\max (s, 1) & \text { if } \ell=0, \\ \max \left(e^{\ell s}, 1\right) & \text { elsewhere. }\end{cases}
$$

Proof. It is clear that Theorem 4 adapts to the case where $\Omega$ is an hypercube, say $(0,1)^{\mathrm{N}}$, if Assumption 5 is strengthened by assuming that also the functions

$$
(x, t, \alpha) \mapsto \frac{1+\ell_{i}}{1-x_{i}}+\frac{b \cdot e_{i}}{\frac{1}{2}\left|\sigma^{T} e_{i}\right|^{2}}
$$

are bounded from above in the set $\{x \in \Omega: d(x) \leq \rho\} \times[0, T] \times \mathcal{A}$, for all $i=1, \cdots$, N. Now the Cauchy problem can be reduced to a problem in the bounded set $(0,1)^{\mathrm{N}}$ by means of a smooth change of variables

$$
\begin{gathered}
\xi(x)=\left(\xi_{1}\left(x_{1}\right), \cdots \xi_{\mathrm{N}}\left(x_{\mathrm{N}}\right)\right): \\
\xi_{i}\left(x_{i}\right)= \begin{cases}e^{\lambda_{i} x_{i}} & \text { if } \lambda_{i} x_{i}<\log \frac{1}{4}, \\
\text { is a polynomial of } x_{i} & \text { if } \log \frac{1}{4} \leq \lambda_{i} x_{i} \leq \log \frac{3}{4}, \\
1-e^{-\lambda_{i} x_{i}} & \text { if } \lambda_{i} x_{i}>\log \frac{3}{4} .\end{cases}
\end{gathered}
$$


Omitting the dependence by $t$ and $\alpha$, the new volatility and drift are

$$
\begin{aligned}
& \tilde{\sigma}_{i j}(\xi)=\lambda_{i} \xi_{i} \sigma_{i j}(x(\xi)), \\
& \tilde{b}_{i}(\xi)=\lambda_{i} \xi_{i} b_{i}(x(\xi))+\frac{1}{2}\left(\lambda_{i} \xi_{i}\left|\sigma^{T}(x(\xi)) e_{i}\right|\right)^{2},
\end{aligned}
$$

near at $\xi_{i}=0$, while near at $\xi_{i}=1$ we have

$$
\begin{aligned}
& \tilde{\sigma}_{i j}(\xi)=\lambda_{i}\left(1-\xi_{i}\right) \sigma_{i j}(x(\xi)), \\
& \tilde{b}_{i}(\xi)=\lambda_{i}\left(1-\xi_{i}\right) b_{i}(x(\xi))+\frac{1}{2}\left(\lambda_{i}\left(1-\xi_{i}\right)\left|\sigma^{T}(x(\xi)) e_{i}\right|\right)^{2} .
\end{aligned}
$$

After easy computations, Assumption 6 and hypothesis (3.4) guarantee that the problem obtained after the change of variables satisfies the needed hypotheses with blow-up parameter given by $\ell_{i} / \lambda_{i}$. Thus the thesis follows by Theorem 4 .

In the linear (uncontrolled) case, the well-posedness of Cauchy problems with unbounded coefficients have been extensively study: we mention, for instance, [17] and [14], both making use of semigroup approach, or [8]. The paper [17] deals with the problem with $\sigma=\sigma(x)>\omega(x) I$ (with $\omega$ a positive function of $x$ ), $b=b(x)$, $f=0, r=0$, and $u_{o}$ bounded. It establishes that the Cauchy problem is well posed in the class of bounded functions, if a related elliptic equation (the so called resolvent equation) has an unbounded supersolution in the set $\{|x| \geq R\}$. Under our hypothesis (3.1), the desired supersolution can be easily found, nevertheless our Theorem 5 provides a larger uniqueness class, and can handle degenerate ellipticity and unbounded data. Of course, the result in [17] holds also when our structural condition (3.1) fails.

\section{Proof of the strong comparison principle}

We give the proof of comparison principle in full details only for Theorem 4: the case with smooth domain is simpler, and it is outlined at the end of this Section. To begin with, we produce a barrier function.

Lemma 1 Under Assumption 4, for every $\bar{x} \in \partial \Omega$ there exist a neighborhood $N$ of $\bar{x}$ and a function $g \in \mathcal{C}^{2}(\Omega \cap N) \cap \mathcal{C}(\Omega \cap \bar{N})$ so that

$$
\begin{aligned}
g>0 & \text { in } \Omega \cap N, \\
\mathcal{L}_{t \alpha} g \geq 0 & \text { in } \Omega \cap N,
\end{aligned}
$$

for all $t \in[0, T], \alpha \in \mathcal{A}$, and

$$
g(x) \rightarrow+\infty \quad \text { as } x \rightarrow \hat{x},
$$

for all $\hat{x} \in \partial \Omega \cap N$. 
If, in addition, Assumption 5 holds, we can chose $N$ independent from $\bar{x} \in$ $\partial \Omega$, actually $N=\{x: d(x)<\rho\}$ with $\rho>0$, so that (4.1) and (4.2) are satisfied and

$$
g(x)=O\left(\sum_{i=1}^{\mathrm{N}} h_{\ell_{i}}\left(x_{i}\right)\right) \quad \text { as } x \rightarrow \hat{x}
$$

for all $\hat{x} \in \partial \Omega$.

Proof. Let $N$ be an hypercube centered at $\bar{x}$, and let $\rho$ its half-hedge. By (2.5) we can chose $\rho$ as small as $\mathrm{I}(\hat{x}) \subset \mathrm{I}(\bar{x})$ for all $\hat{x} \in \partial \Omega \cap N$ and

$$
\mathrm{C}_{i}:=\sup \left\{\frac{1}{x_{i}}-\frac{b \cdot e_{i}}{\frac{1}{2}\left|\sigma^{T} e_{i}\right|^{2}}: x \in \Omega \cap N, t \in[0, T], \alpha \in \mathcal{A}\right\}<\infty
$$

for all $i \in \mathrm{I}(\bar{x})$. Hence we take

$$
g(x)=\sum_{i \in \mathrm{I}(\bar{x})} \int_{x_{i}}^{\rho} e^{-\mathrm{C}_{i} \xi} \xi^{-1} d \xi
$$

Properties (4.1) and (4.3) hold trivially. In addition we get after computations that

$$
\mathcal{L}_{t \alpha} g(x)=\sum_{i \in \mathrm{I}(\bar{x})} \frac{1}{2}\left|\sigma^{T} e_{i}\right|^{2} e^{-\mathrm{C}_{i} x_{i}} x^{-1}\left(\mathrm{C}_{i}+\frac{b \cdot e_{i}}{\frac{1}{2}\left|\sigma^{T} e_{i}\right|^{2}}-\frac{1}{x_{i}}\right) \geq 0
$$

by construction.

Besides, if also Assumption 3 is fulfilled, we can take $N=\{x: d(x)<\rho\}$ in such a way that

$$
\mathrm{C}_{i}:=\max \left(1, \sup \left\{\frac{1+\ell_{i}}{x_{i}}-\frac{b \cdot e_{i}}{\frac{1}{2}\left|\sigma^{T} e_{i}\right|^{2}}: x \in \Omega \cap N, t \in[0, T], \alpha \in \mathcal{A}\right\}\right)
$$

is finite for all $i=1, \cdots, \mathrm{N}$ and for suitable $\ell_{i} \geq 0$. Hence we take

$$
g(x)=\sum_{i=1}^{\mathrm{N}} \int_{x_{i}}^{+\infty} e^{-\mathrm{C}_{i} \xi} \xi^{-1-\ell_{i}} d \xi
$$

Properties (4.1) and (4.2) hold as before. Concerning (4.4), it is trivial that $g / \sum h_{\ell_{i}}$ is bounded in $\Omega \cap N$.

We are now ready to prove the comparison result.

Proof of Theorem 4. We begin by assuming ab absurdo that

$$
M:=\sup \{u(x, t)-v(x, t): x \in \Omega, t \in[0, T)\}>0 .
$$


In order to avoid that maximizing sequences could diverge, we consider for any $\beta>0$ the function

$$
\Psi_{\beta}(x, t):=u(x, t)-v(x, t)-\beta\left(1+|x|^{2}\right),
$$

defined for any $x \in \Omega, t \in[0, T)$, and set

$$
M_{\beta}:=\sup \left\{\Psi_{\beta}(x, t): x \in \Omega, t \in[0, T)\right\} .
$$

By hypothesis (2.9) (respectively, (2.10) if we are dealing with item (ii)), for every $\beta>0$ there exists $R_{\beta}$ such that

$$
M_{\beta}=\sup \left\{\Psi_{\beta}(x, t): x \in \Omega,|x|<R_{\beta}, t \in[0, T)\right\} .
$$

This in turn allows to assume w.l.g. that every maximizing sequence for $\Psi_{\beta}$ converges. In addition, standard arguments give that

$$
M_{\beta} \rightarrow M \quad \text { as } \beta \rightarrow 0 .
$$

By now on, we shall argue for a particular $\beta>0$ fulfilling

$$
M_{\beta}>0 .
$$

We distinguish two alternating situations:

I) The function $\Psi_{\beta}$ admits a maximum point $(\hat{x}, \hat{t}) \in \Omega \times[0, T]$,

II) For every sequence $\left(x_{n}, t_{n}\right)$ which maximizes the function $\Psi_{\beta}$, there exists $(\bar{x}, \bar{t}) \in \partial \Omega \times[0, T]$ so that $\left(x_{n}, t_{n}\right) \rightarrow(\bar{x}, \bar{t})$ as $n \rightarrow \infty$.

The case I) is rejected by the same arguments of [16], because $\sigma$ and $b$ are Lipschitz continuous in the interior of $\Omega$.

We come to show that also II) brings a contradiction. To this end, we chose once and for all a maximizing sequence $\left(x_{n}, t_{n}\right)$ converging to a point $(\bar{x}, \bar{t}) \in$ $\partial \Omega \times[0, T]$, and we introduce the auxiliary function:

$$
\Psi_{\delta}(x, t):=\Psi_{\beta}(x, t)-\delta g(x)=u(x, t)-v(x, t)-\left[\beta\left(1+|x|^{2}\right)+\delta g(x)\right],
$$

defined for every $x \in \Omega \cap \bar{N}$ and $t \in[0, T]$. Here $N$ and $g$ (possibly depending on $\bar{x})$ have been produced in Lemma 1 . For every $\delta>0$, we set

$$
M_{\delta}:=\sup \left\{\Psi_{\delta}(x, t): x \in \Omega \cap \bar{N}, t \in[0, T]\right\} .
$$

We summarize some useful properties of the functions $\Psi_{\delta}$ in a Lemma, that shall be proved later on.

Lemma 2 There is a sequence of vanishing parameters, that we still denote by $\delta$, such that the following holds. For every $\delta>0$, the function $\Psi_{\delta}$ achieves its maximum at a point $\left(x_{\delta}, t_{\delta}\right)$, where $x_{\delta}$ belongs to the interior of $\Omega \cap N$ and $t_{\delta}<T$. Moreover

$$
M_{\delta} \rightarrow M_{\beta} \quad \text { and } \quad \delta g\left(x_{\delta}\right) \rightarrow 0 \quad \text { as } \delta \rightarrow 0 .
$$


We are now ready to double the $x$ variable by considering for any $\varepsilon>0$ the function

$$
\begin{array}{r}
\Psi_{\delta \varepsilon}(x, y, t):=u(x, t)-v(y, t)-\left[\beta\left(1+\frac{1}{2}\left(|x|^{2}+|y|^{2}\right)\right)+\delta g(x)\right. \\
\left.+\frac{1}{2 \varepsilon}|x-y|^{2}\right]
\end{array}
$$

defined for all $x, y \in \Omega \cap \bar{N}$ and $t \in[0, T]$. The proof of next Lemma is completely standard.

Lemma 3 There is a sequence of vanishing parameters, that we still denote by $\varepsilon$, such that the following holds. For any $\delta$ and $\varepsilon$, the function $\Psi_{\delta \varepsilon}$ achieves maximum. If $\left(x_{\delta \varepsilon}, y_{\delta \varepsilon}, t_{\delta \varepsilon}\right)$ stands for a maximum point, we have

$$
\begin{gathered}
\frac{1}{2 \varepsilon}\left|x_{\delta \varepsilon}-y_{\delta \varepsilon}\right|^{2} \rightarrow 0, \quad\left(x_{\delta \varepsilon}, y_{\delta \varepsilon}, t_{\delta \varepsilon}\right) \rightarrow\left(x_{\delta}, x_{\delta}, t_{\delta}\right), \\
u\left(x_{\delta \varepsilon}, t_{\delta \varepsilon}\right)-v\left(y_{\delta \varepsilon}, t_{\delta \varepsilon}\right) \rightarrow M_{\delta}+\beta\left(1+\left|x_{\delta}\right|^{2}\right)+\delta g\left(x_{\delta}\right),
\end{gathered}
$$

as $\varepsilon \rightarrow 0$.

Combining Lemmas 2 and 3, we may assume without loss of generality that $\left(x_{\delta \varepsilon}, y_{\delta \varepsilon}, t_{\delta \varepsilon}\right)$ is a maximum point for the function $\Psi_{\delta \varepsilon}$ in a relative open subset of $\Omega \times \Omega \times[0, T)$, and that there is a sequence of positive numbers $s=s(\delta)$ so that

$$
x_{\delta \varepsilon}, y_{\delta \varepsilon} \in\{x \in \Omega: d(x) \geq s\} \quad \text { and } \quad t_{\delta \varepsilon} \leq T-s<T \quad \text { for all } \varepsilon>0 .
$$

Applying [6, Theorem 8.4] gives that, for all $\varepsilon>0$, there are $\tau_{1}, \tau_{2} \in \mathbb{R}$ and two matrices $X, Y$ such that

$$
\begin{gathered}
\left(\tau_{1}, \frac{1}{\varepsilon}(x-y)+\delta D g(x)+\beta x, X+\delta D^{2} g(x)+\beta I\right) \in \overline{\mathcal{P}}^{2,+} u(x, t), \\
\left(\tau_{2}, \frac{1}{\varepsilon}(x-y)-\beta y, Y-\beta I\right) \in \overline{\mathcal{P}}^{2,-} v(y, t), \\
\tau_{1}-\tau_{2}=0, \quad\left(\begin{array}{cc}
X & 0 \\
0 & -Y
\end{array}\right) \leq 3 \varepsilon\left(\begin{array}{cc}
I & -I \\
-I & I
\end{array}\right) .
\end{gathered}
$$

Here and in the following, we omit the dependence by $\delta$ and $\varepsilon$ for simplicity of notations. Afterward, since $u$ and $v$ are respectively sub/supersolution of (1.3) in viscosity sense, there is a sequence of controls $\alpha_{k}$ such that

$$
\begin{aligned}
-\frac{1}{2}(\operatorname{tr} & {\left.\left[\sigma \sigma^{T}(x) X\right]-\operatorname{tr}\left[\sigma \sigma^{T}(y) Y\right]\right)-\frac{1}{\varepsilon}(x-y)(b(x)-b(y)) } \\
-\beta & \left(\frac{1}{2}\left(\operatorname{tr}\left[\sigma \sigma^{T}(x)\right]-\operatorname{tr}\left[\sigma \sigma^{T}(y)\right]\right)+b(x) \cdot x-b(y) \cdot y\right) \\
- & f(x)+f(y)+r(x) u(x)-r(y) v(y, t)+\delta \mathcal{L}_{t \alpha} g(x) \leq \frac{1}{k}
\end{aligned}
$$


for any integer $k$. Here all the functions are computed at $t=t_{\varepsilon \delta}$ and $\alpha=\alpha_{k}$. Hence the same computations of [6, Example 3.6] yield

$$
r(x)[u(x)-v(y)]+\delta \mathcal{L}_{t \alpha} g(x) \leq \frac{1}{k}+\mathrm{C}(1+\beta)\left((1+\mathrm{A})|x-y|+\frac{1}{\varepsilon}|x-y|^{2}\right)
$$

where $\mathrm{A}=\mathrm{A}(\delta)=\sup \{|v(y, t)|: y \in \overline{\{x \in \Omega: d(x) \geq s\} \cap N}, t \in[0, T-s]\}$, and $\mathrm{C}=\mathrm{C}(\delta)$ only depends on the Lipschitz constant of $\sigma, b, f$ on $\{x \in \Omega: d(x) \geq s\}$. Now the second term in the left-hand side is greater or equal to zero by (4.2). In addition we may suppose without loss of generality that $r \geq 1$ : otherwise, it suffices to multiply both $u$ and $v$ by $\exp ((\inf r-1)(T-t))$. Then extracting the limit with respect to $\varepsilon \rightarrow 0$ and recalling Lemma 3 yields

$$
M_{\delta}+\left(1+\beta\left|x_{\delta}\right|^{2}\right)+\delta g\left(x_{\delta}\right) \leq 1 / k
$$

for all $k$. Hence

$$
M_{\delta} \leq 0 .
$$

Eventually sending $\delta \rightarrow 0$ and using Lemma 2 we get

$$
M_{\beta} \leq 0,
$$

which contradicts (4.5).

We next prove Lemma 2, for the sake of completeness.

Proof of Lemma 2. First of all we take $\left(x_{n}, t_{n}\right)$ a maximizing sequence for $\Psi_{\beta}$; since we are standing in case II), we have that $\left(x_{n}, t_{n}\right) \rightarrow(\bar{x}, \bar{t})$ with $\bar{x} \in \partial \Omega$, as $n \rightarrow \infty$. Next we take the neighborhood $N$ of $\bar{x}$ and the function $g: \Omega \cap \bar{N} \rightarrow \mathbb{R}$ produced in Lemma 1, and we introduce the function

$$
\Psi_{\delta}:(\Omega \cap \bar{N}) \times[0, T] \rightarrow \mathbb{R}, \quad \Psi_{\delta}(x, t)=\Psi_{\beta}(x, t)-\delta g(x),
$$

with

$$
\delta=\delta(n)=1 / n g\left(x_{n}\right) .
$$

Because $x_{n} \rightarrow \bar{x} \in \partial \Omega$, property (4.3) (resp., (4.4)) ensures that $g\left(x_{n}\right) \rightarrow \infty$ and therefore $\delta \rightarrow 0$ as $n \rightarrow \infty$.

By standard semicontinuity arguments, (2.9), and (4.3) (resp., (2.10), (2.11), and (4.4)) ensures that $\Psi_{\delta}$ admits a global maximum, say $\left(x_{\delta}, t_{\delta}\right)$, in the set $(\Omega \cap \bar{N}) \times$ $[0, T]$. By construction

$$
M_{\beta} \geq M_{\delta} \geq \Psi_{\delta}\left(x_{n}, t_{n}\right)=\Psi_{\beta}\left(x_{n}, t_{n}\right)-1 / n,
$$

hence $M_{\delta} \rightarrow M_{\beta}$. In addition, by the inequalities

$$
M_{\beta} \geq \Psi_{\beta}\left(x_{\delta}, t_{\delta}\right)=M_{\delta}+\delta g\left(x_{\delta}\right) \geq M_{\delta}
$$


it follows that $\delta g\left(x_{\delta}\right) \rightarrow 0$ and $\Psi_{\beta}\left(x_{\delta}, t_{\delta}\right) \rightarrow M_{\beta}$. This in turn allows to suppose that $t_{\delta}<T$ for all $\delta$. To check this fact, we assume ab absurdo that $t_{\delta} \equiv T$ definitely. Then $\Psi_{\beta}\left(x_{\delta}, T\right) \leq-\beta\left(1+\left|x_{\delta}\right|^{2}\right)$ by (2.8) and, passing to the limit, we get $M_{\beta} \leq-\beta<0$, which conflicts with (4.5).

It remains to check that $x_{\delta}$ do not belong to $\Omega \cap \partial N$. To this end we recall that $\left(x_{\delta}, t_{\delta}\right)$ is a maximizing sequence for $\Psi_{\beta}$, then $x_{\delta} \rightarrow \hat{x} \in \partial \Omega$ as we are standing in item II). This complete the proof if the additional Assumption 5 holds, because in this case $\partial \Omega \subset N$. Otherwise hypothesis (2.11) allows to suppose that the function $\Psi_{\beta}$ achieves a strict maximum at the point $(\bar{x}, \bar{t})$ : if not, it suffices to replace $\Psi_{\beta}(x, t)$ with $\Psi_{\beta}(x, t)-|x-\bar{x}|^{4}-|t-\bar{t}|^{2}$. Hence $x_{\delta} \rightarrow \bar{x} \in N$ and the proof is completed.

Finally we sketch the proof in the smooth case. The proof of Theorem 2 relies on the same arguments, but there is no need of the auxiliary function $\Psi_{\beta}$, because the domain is pre-compact. So one can set $\beta=0$ from the beginning and follow the line of the proof of Theorem 4. The needed barrier function $g$ is given by next Lemma.

Lemma 4 Under Assumption 2, for every $\bar{x} \in \partial \Omega$ there exist a neighborhood $N$ of $\bar{x}$ and a function $g \in \mathcal{C}^{2}(\Omega \cap N) \cap \mathcal{C}(\Omega \cap \bar{N})$ so that

$$
\begin{aligned}
g>0 & \text { in } \Omega \cap N, \\
\mathcal{L}_{t \alpha} g \geq 0 & \text { in } \Omega \cap N,
\end{aligned}
$$

for all $t \in[0, T], \alpha \in \mathcal{A}$, and

$$
g(x) \rightarrow+\infty \quad \text { as } x \rightarrow \hat{x},
$$

for all $\hat{x} \in \partial \Omega \cap N$.

If, in addition, Assumption 3 holds, we can chose $N$ independent from $\bar{x} \in \partial \Omega$, actually $N=\{x: d(x)<\rho\}$ with $\rho>0$, so that (4.7) and (4.8) are satisfied and

$$
g(x)=O\left(h_{\ell}(d)\right) \quad \text { as } x \rightarrow \hat{x},
$$

for all $\hat{x} \in \partial \Omega$.

Proof. First, let $N$ be a ball centered at $\bar{x}$, and $\rho$ its radius. By hypothesis we can chose $\rho$ so small that the function $d$ is of class $\mathcal{C}^{2}$ in $\Omega \cap N$ and

$$
\mathrm{C}:=\sup \left\{\frac{1}{d}+\frac{\mathcal{L}_{t, \alpha} d}{\frac{1}{2}\left|\sigma^{T} D d\right|^{2}}: x \in \Omega \cap N, t \in[0, T], \alpha \in \mathcal{A}\right\}<\infty .
$$

Hence we take

$$
g(x)=\int_{d(x)}^{\rho} e^{-\mathrm{C} \xi} \xi^{-1} d \xi
$$

Properties (4.7)-(4.9) are trivially satisfied. 
Besides, under Assumption 3, we may take $\rho>0$ and $\ell \geq 0$ so that the function $d$ is of class $\mathcal{C}^{2}$ in $\{x \in \Omega: d(x)<\rho\}$, and

$$
\mathrm{K}:=\sup \left\{\frac{1+\ell}{d}+\frac{\mathcal{L}_{t, \alpha} d}{\frac{1}{2}\left|\sigma^{T} D d\right|^{2}}: x \in \Omega, d(x)<\rho, t \in[0, T], \alpha \in \mathcal{A}\right\}<\infty .
$$

So the desired auxiliary function is

$$
g(x)=\int_{d(x)}^{\rho} e^{-\mathrm{K} \xi} \xi^{-1-\ell} d \xi,
$$

for all $x \in \Omega$ with $d(x)<\rho$.

\section{References}

[1] M. BARDI and I. CAPUZZO-DOLCETTA, Optimal control and viscosity solutions of Hamilton-Jacobi-Bellman equations. Systems and Control: Foundations and Applications, Boston, MA: Birkhäuser, 1997.

[2] T. BJÖRK and A. GOMBANI, Minimal realizations in interest rate models, Finance Stoch. 3(4) (1999), 413-432.

[3] M. CAMPITI, G. METAFUNE, and D. PALLARA, One-dimensional Feller semigroups with reflecting barriers, J. Math. Anal. Appl. 244(1) (2000), 233-250.

[4] F. H. CLARKE, Optimization and nonsmooth analysis. Reprint, Classics in Applied Mathematics 5, Philadelphia, PA: SIAM, 1990.

[5] J. C. COX, J. E. INGERSOLL, and S. A. ROSS, An intertemporal general equilibrium model of asset prices, Econometrica 53 (1985), 363-384.

[6] M. G. CRANDALL, H. ISHII, and P.-L. LIONS, User's guide to viscosity solutions of second order partial differential equations, Bull. Am. Math. Soc., New Ser. 27(1) (1992), 1-67.

[7] M. G. CRANDALL, R. T. NEWCOMB, and Y. TOMITA, Existence and uniqueness for viscosity solutions of degenerate quasilinear elliptic equations in $R^{N}$, Appl. Anal. 34(1/2) (1989), 1-23.

[8] G. DA PRATO and B. GOLDYS, Elliptic operators on $\mathbf{R}^{d}$ with unbounded coefficients, J. Differ. Equations 172(2) (2002), 333-358.

[9] W. FELLER, Two singular diffusion problems, Ann. of Math. (2) 54 (1951), $173-182$.

[10] W. FELLER, The parabolic differential equations and the associated semigroups of transformations, Ann. of Math. (2) 55 (1952), 468-519. 
[11] W. FELLER, Diffusion processes in one dimension, Trans. Amer. Math. Soc. 77 (1954), 1-31.

[12] W. H. FLEMING and H. SONER, Controlled Markov processes and viscosity solutions, 25 of Applications of Mathematics, Springer-Verlag, New York, 1993.

[13] H. ISHII and P.-L. LIONS, Viscosity solutions of fully nonlinear second-order elliptic partial differential equations, J. Differential Equations 83(1) (1990), $26-78$.

[14] S. KAMIN, R. KERSNER, and A. TESEI, On the Cauchy problem for a class of parabolic equations with variable density, Atti Accad. Naz. Lincei Cl. Sci. Fis. Mat. Natur. XI Ser. Rend. Lincei Mat. Appl. 9(4) (1998), 279-298.

[15] D. LAMBERTON and B. LAPEYRE, Introduction to stochastic calculus applied to finance, (Introduction au calcul stochastique appliqué à la finance.) 2ème éd., Paris: Ellipses 176, 1997.

[16] P.-L. LIONS, Optimal control of diffusion processes and Hamilton-JacobiBellman equations. II: Viscosity solutions and uniqueness, Commun. Partial Differ. Equations 8(11) (1983), 1229-1276.

[17] G. METAFUNE, D. PALLARA, and M. WACKER, Feller semigroups on $\mathbf{R}^{N}$, Semigroup Forum 65(2) (2002), 159-205.

[18] J. Y. YONG and X. ZHOU, Stochastic controls, Hamiltonian systems and HJB equations, New York, NY: Springer, 1999.

Received 13 January 2006; accepted 4 December 2006

To access this journal online: http://www.birkhauser.ch 